\title{
Breast cancer therapy affects the expression of antineonatal Nav1.5 antibodies in the serum of patients with breast cancer
}

\author{
HARISHINI RAJARATINAM ${ }^{1}$, NUR SYAHMINA RASUDIN ${ }^{1}$, TENGKU AHMAD DAMITRI AL ASTANI ${ }^{2,3}$, \\ NOOR FATMAWATI MOKHTAR ${ }^{4}$, MAYA MAZUWIN YAHYA ${ }^{5}$, WAN ZAINIRA WAN ZAIN ${ }^{5}$, \\ NURUL ASMA-ABDULLAH ${ }^{1}$ and WAN EZUMI MOHD FUAD ${ }^{1}$ \\ ${ }^{1}$ School of Health Sciences; ${ }^{2}$ Department of Chemical Pathology, School of Medical Sciences, Health Campus, \\ Universiti Sains Malaysia (USM); ${ }^{3}$ Breast Cancer Awareness and Research (BestARi) Unit, \\ Hospital Universiti Sains Malaysia (HUSM); ${ }^{4}$ Institute for Research in Molecular Medicine (INFORMM); \\ ${ }^{5}$ Department of Surgery, School of Medical Sciences, Health Campus, \\ Universiti Sains Malaysia (USM), Kubang Kerian, Kelantan 16150, Malaysia
}

Received August 11, 2020; Accepted November 5, 2020

DOI: $10.3892 / \mathrm{ol} .2020 .12369$

\begin{abstract}
Neonatal Nav1.5 (nNav1.5) is the alternative splice variant of Nav1.5 and it has been widely associated with the progression of breast cancer. The immunological context of nNav1.5 with respect to breast cancer metastases remains unexplored. The presence of antibodies against nNav1.5 may highlight the immunogenicity of nNav1.5. Hence, the aim of the present study was to detect the presence of antineonatal Nav1.5 antibodies (antinNav1.5-Ab) in the serum of patients with breast cancer and to elucidate the effects of breast cancer therapy on its expression. A total of 32 healthy female volunteers and 64 patients with breast cancer were randomly recruited into the present study as the control and breast cancer group, respectively. Patients with breast cancer were divided equally based on their pre- and ongoing-treatment status. Serum samples were tested with in-house indirect enzyme-linked immunosorbent assay (ELISA) to detect antinNav1.5-Ab, CD25 (T regulatory cell marker) using an ELISA kit and Luminex assay to detect the expression of metastasis-associated cytokines, such as vascular endothelial growth factor (VEGF), interleukin (IL)-6, IL-10, IL-8, chemokine (C-C motif) ligand 2 and tumor necrosis factor-alpha (TNF- $\alpha$ ) The mean difference in the expression of antinNav1.5-Ab among the three groups (control, pretreatment and ongoing-treatment) was significant $(\mathrm{P}=0.0005)$ and the pretreatment breast cancer group exhibited the highest expression. The concentration of CD25 was
\end{abstract}

Correspondence to: Dr Wan Ezumi Mohd Fuad, School of Health Sciences, Health Campus, Universiti Sains Malaysia (USM), 15 Jalan Raja Perempuan Zainab II, Kubang Kerian, Kelantan 16150, Malaysia

E-mail: wanezumi@usm.my

Key words: antibodies, breast cancer, breast cancer therapy, immune system, metastasis, neonatal Nav1.5 highest in the pretreatment breast cancer group compared with the control and ongoing-treatment groups. There was a significant positive correlation between antinNav1.5-Ab and IL-6 in the pretreatment group $(\mathrm{r}=0.7260 ; \mathrm{P}=0.0210)$ and a significant negative correlation between antinNav1.5-Ab and VEGF in the ongoing-treatment group $(\mathrm{r}=-0.842$; $\mathrm{P}$-value $=0.0040)$. The high expression of antinNav1.5-Ab in the pretreatment group was in accordance with the uninterrupted presence of metastasis and highlighted the immunogenicity of nNav1.5 whereas the low expression of antinNav1.5-Ab in the ongoing-treatment group reflected the efficacy of breast cancer therapy in eliminating metastases. The augmented manifestation of $\mathrm{T}$ regulatory cells in the pretreatment group highlighted the functional role of nNav1.5 in promoting metastasis. The parallel expression of antinNav1.5-Ab with the imbalanced expression of cytokines promoting metastasis (IL-8, IL-6 and TNF- $\alpha$ ) and cytokines that prevent metastasis (IL-10) indicated the role of nNav1.5 in breast cancer growth. The expression of antinNav1.5-Ab in accordance to the metastatic microenvironment indicates the immunogenicity of the protein and highlights the influence of breast cancer therapy on its expression level.

\section{Introduction}

In 2018, breast cancer was one of the leading causes of death for women worldwide, apart from other types of cancer, such as lung and colorectal cancer (1). Of the most resourceful organizations that have updated breast cancer data are the World Health Organization's International Agency for Research on Cancer (IARC) and the Centers for Disease Control and Prevention (CDC). In 2018, IARC compiled a brief study known as GLOBOCAN 2018 (1) that highlighted the prevalence, mortality and incidence rates of different cancer forms. According to the aforementioned study, breast cancer has the highest incidence $(24.2 \%)$, mortality $(15.0 \%)$, and prevalence $(30.1 \%)$ rates among female patients worldwide (1). GLOBOCAN 2018 also reported that Asia has the most significant number of breast cancer cases, followed by 
Europe and North America (1). According to a report released by the CDC in 2019, there has been no change in the incidence of breast cancer acquisition over the last decade (2). In spite this, the trend has risen for black, Asian and Pacific Islander women (2).

Breast cancer occurs when healthy breast cells begin to develop rapidly without the completion of their regular cell cycle (3). Much of the initial cancer growth occurs within the breast lobules and ducts (2) However, the progression of cancer development can cause cancer to spread beyond the breast $(4,5)$. Such an occurrence is called metastasis (6) Metastasis is caused by the dissociation of cancer cells from the primary tumor site, accompanied by the breakdown of the extracellular membrane allowing the cancer cells to enter the bloodstream or lymphatic vessels, creating a secondary tumor in other parts of the body which includes primary organs, such as lungs and liver $(6,7)$.

The presence of voltage-gated sodium channels (VGSCs) has been identified over the last decade to increase the progression of metastases $(8,9)$. The functional overexpression of VGSCs has been documented in different types of carcinomas (cancers of epithelial origin), such as breast, ovarian and cervical cancers (10-17). The VGSC structure consists of one $\alpha$-subunit (pore-forming) and smaller $\beta$-subunits (10). There is a total of 9 separate subunits of $\alpha$ (Nav1.1-Nav1.9) and four $\beta$ subunits $(\beta 1-\beta 4)(10)$. The neonatal alternative splice variant of Nav1.5 (nNav1.5) has been widely recognized as one of the culprits in cancerous breast cells that metastasize (10-12). Nav1.5 is encoded by the SCN5A gene and generally suppresses the action mechanism of most sodium channels within the VGSC family (18). The Nav1.5 channel typically carries an inward sodium ion current which depolarizes the membrane potential during a heart attack (19).

The neonatal isoform of Nav1.5 has developed as a result of epigenetic dysregulation through the VGSC $\alpha$ alternative splicing at D1:S3 (20). The upregulation of nNav1.5 in breast cancer is suggestive of onco-foetal gene expression since nNav1.5 would typically be expressed during the foetal stage of human development $(21,22)$. The distinction between Nav1.5 and nNav1.5 in terms of molecular characteristics are the distinguishable 7 amino acid substitutions in the extracellular region and the alternatively spliced exons (D1:S3 5'denotes neonatal Nav1.5 while D1:S3 3'denotes adult Nav1.5) that allow the recognition of these two isoforms $(20,23)$.

The link between Nav1.5 and nNav1.5 with metastasis in breast cancer has been highlighted in several high-quality publications (10-12). The aforementioned studies demonstrated that by enhancing metastasis, functional upregulation of these proteins in breast cancer can contribute to breast cancer progression (10-12). Numerous in vitro studies have suggested that overexpression of these proteins can be observed in highly metastatic breast cancer cells, such as MDA-MB-231 compared with less metastatic breast cancer cell lines, such as MCF-7 (11,23-25). An extensive in vivo study performed by Nelson et al (26) provided a model in 2015, which further solidified that Nav1.5 serves a significant role in fostering breast cancer metastasis. The aforementioned study demonstrated that the downregulation of Nav1.5 expression achieved using lentiviral shRNA led to significantly reduced tumor development, decreased local invasion of the surrounding tissue and mitigated the progression of tumor metastasis to the liver, lungs and spleen in an orthotopic breast tumor mouse model (26). In a clinical study by Fraser et al (12), the expression of nNav1.5 in human breast cancer biopsy sections was significantly upregulated compared with healthy breast tissue. In addition, in the aforementioned study it was demonstrated that the expression of nNav1.5 tested in these tissues of breast cancer was closely associated with metastasis to the lymph nodes (12).

Since metastasis requires blood circulation and lymphatic systems as conventional pathways for progression, it was hypothesized in the present study that the immune system may target the presence of nNav1.5 antigen on the circulating metastasizing cancer cells in the blood system and produce antibodies against it. The evasion of the immune system was identified as one of the 'Hallmarks of Cancer' in 2011 (27), making the investigation of the immune system essential for identification of the existence of antibodies against nNav1.5 and reducing the development of breast cancer metastasis in the present study. To the best of our knowledge, the present study is the first study that aimed to detect the presence of naturally produced antinNav1.5-Ab in the serum of patients with breast cancer. In addition, the present study also elucidated the effect of breast cancer therapy on the expression of antinNav1.5-Ab to provide insight into its role as an immune-surveillance marker in monitoring treatment outcomes. In addition, the expression of $\mathrm{T}$ regulatory (Treg) cells and metastasis-related cytokines, such as interleukin (IL)-6 and vascular endothelial growth factor (VEGF) were investigated in the present study to support and validate the association of antinNav1.5-Ab with breast cancer metastasis.

\section{Materials and methods}

Recruitment of patients. The present study was conducted at the Hospital Universiti Sains Malaysia (HUSM) in collaboration with the USM Breast Cancer Awareness and Research Unit (BestARi) (Kubang Kerian, Malaysia). Ethical approval was granted by the Human Research Ethics Committee of USM (JEPeM) (approval no. USM/JEPeM/18100518). A total of 96 participants were recruited in the present study from March 2019-March 2020. Of these, 32 participants were healthy females (controls), while the remaining 64 participants were patients with breast cancer. These breast cancer patients were classified into two groups ( $n=32$ each) based on their treatment status: Pretreatment (treatments were not performed) and ongoing-treatment (multiple forms of treatment were performed, such as chemotherapy, radiotherapy and surgery). The age range for the subjects in the present study was 23-70 years old. The mean \pm SEM of age for the breast cancer group was $49.160 \pm 2.243$ [95\% confidence interval (CI), 44.58-53.73] whereas for the control group it was $36.59 \pm 1.377$ (95\% CI, 33.78-39.40). The inclusion criteria for patients with breast cancer were as follows: i) Early-invasive or advanced stage breast cancer; ii) no past history of other types of cancer, iii) having received treatments; and iv) no chronic diseases, such as immune disorders, severe diabetes and chronic hypertension. The inclusion criteria for healthy participants were as follows: i) No history of breast cancer, ii) absence of other types of cancer; and no iii) chronic diseases such as immune 
disorders, severe diabetes and chronic hypertension. Written informed consent was obtained from all subjects prior to blood collection.

Serum collection. In total $\sim 3 \mathrm{ml}$ of blood was collected from the participants with the assistance of certified nurses or medical officers. Serum samples were extracted from whole blood by centrifugation at $1,800 \mathrm{x}$ g for $15 \mathrm{~min}$ and stored at $-80^{\circ} \mathrm{C}$.

In-house indirect enzyme-linked immunosorbent assay (ELISA). To the best of our knowledge, there is no commercial ELISA kit available at present for the detection of antinNav1.5-Ab, an optimized in-house indirect ELISA was performed to detect the presence of antibodies produced against nNav1.5 antigen found in the serum. The Nunc Maxisorp ELISA plate (Thermo Fisher Scientific Inc.) was coated with $100 \mu \mathrm{l}$ of nNav1.5 peptide (GenScript Biotech Corp.) working solution $(5 \mu \mathrm{g} / \mathrm{ml})$ and was left to rest at $4^{\circ} \mathrm{C}$ overnight. Following overnight incubation, the plate was washed thrice with PBS. Next, the plate was blocked at $4^{\circ} \mathrm{C}$ with $200 \mu \mathrm{l}$ of $5 \%$ skim milk for $2 \mathrm{~h}$. Following brief washing 3 times, $100 \mu \mathrm{l}$ of serum samples were added at a dilution of 1:400 and the plates were left to rest at $4^{\circ} \mathrm{C}$ overnight. On the third day, the same washing process was repeated with PBS-TWEEN 20 (0.05\%), and the plate was then incubated with $100 \mu \mathrm{l}$ of rabbit anti-human IgG, HRP conjugated secondary antibody (1:5,000; cat. no. CSB-PA00120F1Rb; Cusabio Technology LLC) at $4^{\circ} \mathrm{C}$ for $2 \mathrm{~h}$. Following incubation, the plate was washed thrice with PBS-TWEEN 20. Subsequently, $100 \mu 1$ of 3,3',5,5'-Tetramethylbenzidine (TMB) substrate (Sigma-Aldrich; Merck KgaA) was added to each well for $30 \mathrm{~min}$ at room temperature Sulfuric acid was added to stop the reaction and the absorbances were read spectrophotometrically at $450 \mathrm{~nm}$. The optical density of each sample was scanned using the Varioskan Flash spectral scanning multimode reader (Thermo Fisher Scientific Inc.). Pooled positive and negative sera were used as positive and negative controls, respectively. There was no standard reagent (standard value) available since to the best of our knowledge this is the first time such an assay was developed. All the values were normalized using the blank value.

CD25 sandwich ELISA. A commercial Human Interleukin 2 Receptor $\alpha$ (IL-2R $\alpha /$ CD25) kit (Elabscience) was used according to the manufacturer's instructions. In total, $\sim 100 \mu \mathrm{l}$ of standard and samples were added to the precoated ELISA plate. The plate was precoated with an antibody specific to CD25. In total, $\sim 100 \mu 1$ of biotinylated detection antibodies specific to human CD25 and $100 \mu \mathrm{l}$ of avidin-horseradish peroxidase (HRP) conjugates which were part of the kit were successively added to each microplate well and incubated for $90 \mathrm{~min}$ respectively at $37^{\circ} \mathrm{C}$. Next, the unbound components were washed away, and the substrate was added. The enzyme-substrate reaction was stopped by the addition of $50 \mu \mathrm{l}$ of stop solution. The optical densities were measured spectrophotometrically at a wavelength of $450 \mathrm{~nm}$ using the Varioskan Flash spectral scanning multimode reader (Thermo Fisher Scientific Inc.). The CD25 concentration was calculated using the standard curve.
Magnetic luminex assay. An additional method to indicate the presence of metastases in the serum of the participants was included in the study. A custom-made magnetic Luminex assay kit, Human Magnetic Luminex Screening Assay-10 PLEX (R\&D Systems, Inc.) was produced based on a selection of 10 cytokines. However, only 6 cytokines were selected: Pro-inflammatory cytokines, such as IL-8, IL-6, TNF- $\alpha$, VEGF, chemokine (C-C motif) ligand 2 (CCL2) and antiinflammatory cytokines, such as IL-10. The other four cytokines depicted low expressions below the detection range, thus were not reported here. About 20 serum samples were utilized to represent the control group whereas for pretreatment and ongoing-treatment groups, 10 serum samples were utilized for each group.

The Luminex assay was performed according to the manufacturer's instructions. In brief, $\sim 50 \mu \mathrm{l}$ of standard or sample was pipetted into the respective wells followed by $50 \mu 1$ of diluted microparticle cocktail. Incubation took place in a shaker at $72 \times \mathrm{g}$ for $2 \mathrm{~h}$ at room temperature. After washing, $50 \mu \mathrm{l}$ of the diluted biotin antibody cocktail was added to each well (plate was covered) for $1 \mathrm{~h}$ at room temperature on the shaker at $72 \mathrm{x}$ g. The same washing process was repeated before adding $50 \mu \mathrm{l}$ of diluted streptavidin-PE substrate to each well for $30 \mathrm{~min}$ at room temperature on the shaker at $72 \mathrm{x} \mathrm{g}$. The washing process was repeated, followed by additional washing, which involved adding $100 \mu \mathrm{l}$ of wash buffer to each well at room temperature at $72 \mathrm{x} g$ for $2 \mathrm{~min}$. The plate was read within $1.5 \mathrm{~h}$ using a Luminex ${ }^{\circledR}$ analyzer (Luminex Corporation).

Statistical analysis. GraphPad Prism version 8 (GraphPad Inc.) was used for all the statistical analyses. For all the tests, duplicate biological replicates were performed. Multiple comparisons that were parametric were analyzed using one-way ANOVA followed by the post hoc Tukey's whereas Kruskal-Wallis test was used for non-parametric multiple comparisons followed by the post hoc Dunn's multiple comparison test. Spearman correlation was used for the correlation analysis between cytokines and antinNav1.5-Ab expression. Error bars represent either standard error of mean (SEM) or interquartile range (IQR). The strength of the r-value correlation was interpreted based on a previous study (28): Poor-correlation $(\mathrm{r} \leq 0.25)$; fair-correlation $(\mathrm{r}=0.26-0.50)$; good correlation $(\mathrm{r}=0.51-0.75)$ and excellent correlation $(\mathrm{r}=0.76-1.00)$. Normality of the data (skewness and kurtosis) was determined based on the guidelines by George and Mallery (29) and Schmider et al (30). $\mathrm{P}<0.05$ was considered to indicate a statistically significant difference.

\section{Results}

Optical density of antinNav1.5-Ab in the serum samples. AntinNav1.5-Ab expression in the serum of control, pretreatment and ongoing-treatment groups were compared. Considering the mean expression of antinNav1.5-Ab expression of the control group as a baseline, it was demonstrated that the expression of antinNav1.5-Ab were upregulated in patients with breast cancer regardless of their treatment status. When examining the effect of breast cancer treatment on the antibodies, the expression of antinNav1.5-Ab was higher in 


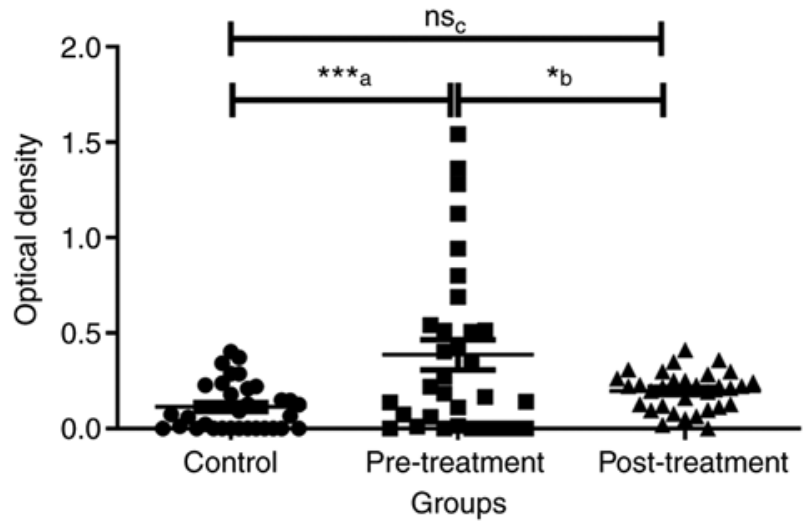

Figure 1. Difference in antinNav1.5-Ab expression in the serum of control, pretreatment and ongoing-treatment patients with breast cancer. A comparison of antinNav1.5-Ab expression levels between the control, pretreatment and ongoing-treatment groups ( $\mathrm{n}=32$ in each group) using one-way ANOVA test and the post hoc Tukey's multiple comparison test. The error bars represent the SEM. Comparison between the means of control and pretreatment groups ( $\mathrm{a} ; \mathrm{P}=0.0004)$. Comparison between the means of pretreatment and ongoing-treatment groups $(\mathrm{b} ; \mathrm{P}=0.0184)$. Comparison between the means of control and ongoing-treatment groups $(c ; P=0.4449) .{ }^{* * *} \mathrm{a}=0.0001-0.0010$; $\mathrm{k} b=0.0100-0.0490$; and ${ }^{\mathrm{ns}} \mathrm{c}=0.4449(\mathrm{P} \geq 0.05)$, ns. NS, not significant; antineonatal Nav1.5 antibodies (antinNav1.5-Ab).

the pretreatment group compared with the ongoing-treatment group (Fig. 1). The mean \pm SEM for control, pretreatment and ongoing-treatment groups were $0.1139 \pm 0.0225,0.3869 \pm 0.0787$ and $0.1973 \pm 0.0180$, respectively (Fig. 1). The optical densities of antinNav1.5- $\mathrm{Ab}$ in the serum of control, pretreatment and ongoing-treatment groups were compared using one-way ANOVA test and the difference between the means of the three groups were significant $(\mathrm{P}=0.0005)$. Further analysis using the post hoc Tukey's test demonstrated that the mean differences between two pairs: Control vs. pretreatment and pretreatment vs. ongoing-treatment were significant (Fig. 1). However, the mean difference between control and ongoing-treatment groups was not significant (Fig. 1).

Concentration of CD25 a Treg cell marker in the serum samples. A commercial sandwich ELISA kit was used to detect the concentration of CD25 in the serum samples of control, pre-treatment and ongoing-treatment groups. Concentrations of unknown samples were interpreted from the standard curve plotted based on the known concentrations of the standards (Fig. 2). The standard curve presented was used to calculate the actual concentration of CD25 by inserting the optical densities into the equation. The concentrations of CD25 in the serum of control, pretreatment, and ongoing-treatment groups were compared using the Kruskal Wallis test and the median differences between these groups were not significant $(\mathrm{P}=0.6390)$. However, based on the median comparison of the CD25 concentrations between the three groups, it was evident that the expression of $\mathrm{T}$ reg cells was increased in the pretreatment group and reduced in the ongoing-treatment group (Fig. 3).

Expression of cytokines associated with breast cancer metastasis. Upregulation and downregulation of cytokines in the pretreatment and ongoing-treatment groups were determined by considering the cytokine profile of the control

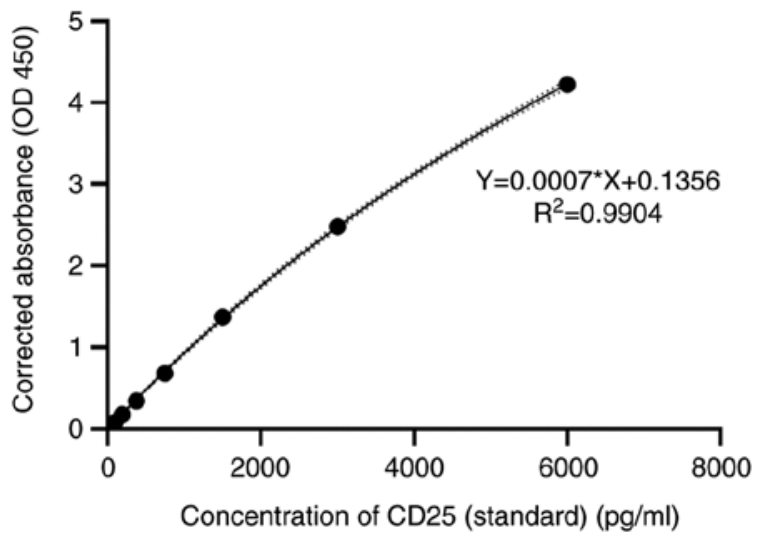

Figure 2. Standard curve for CD25 concentration. Standard curve of CD25 absorbance (obtained using sandwich ELISA) plotted against known concentrations used to interpolate the unknown concentrations of the samples. OD, optical density.

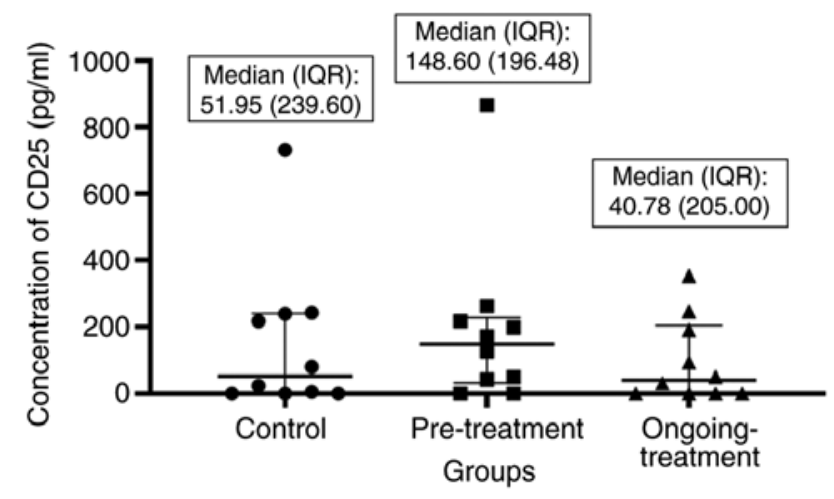

Figure 3. Differences in the expression of CD25 in the serum of control, pretreatment and ongoing-treatment groups. Comparison of CD25 concentration levels between the control, pretreatment and ongoing-treatment groups using Kruskal Wallis test $(\mathrm{P}=0.6390)$. The error bars represent the interquartile range. IQR, interquartile range.

as a baseline. The pretreatment group exhibited the highest concentration of IL-8 compared with the other two groups (Table I). There were minute differences in the concentration levels of VEGF and CCL2 cytokines among the three groups (Table I). The control group exhibited the highest concentration of VEGF and CCL2 followed by the pretreatment group. As anticipated, the ongoing-treatment group had the lowest concentrations of VEGF and CCL2 compared with the other two groups (Table I).

Comparison of net median fluorescence intensity (MFI) values of $I L-6, I L-10$ and $T N F-\alpha$ cytokines in the serum samples. MFI values were used to analyze the trend between three study groups, as the concentration of the analytes in the samples were relatively low (Table II). A similar method has also been published, which supports the use of MFI as valuable data for statistical analysis (31). The serum samples from patients with breast cancer in the pretreatment group had a higher intensity of IL- 6 and TNF- $\alpha$ compared with the other groups. However, the downregulation of IL-6 and TNF- $\alpha$ in the ongoing-treatment group was notable as the serum samples from this group exhibited the lowest expression of IL-6, IL-10 
Table I. Concentration of IL-8, VEGF and CCL2 in control ( $\mathrm{n}=20)$, pretreatment and ongoing-treatment groups ( $\mathrm{n}=10$ each).

\begin{tabular}{lccrr}
\hline Cytokines & Control & Pretreatment & Ongoing-treatment & P-value \\
\hline IL-8 & $4.074 \pm 0.392$ & $8.557 \pm 2.269$ & $5.159 \pm 0.594$ & $0.0490^{\mathrm{a}}$ \\
VEGF & $70.33 \pm 12.07$ & $64.59 \pm 18.65$ & $60.75 \pm 13.89$ & 0.9250 \\
CCL2 & $269.8 \pm 30.08$ & $249.6 \pm 34.53$ & $240.7 \pm 31.13$ & 0.8020
\end{tabular}

Data values are expressed as mean \pm SEM. Kruskal Wallis test was conducted to compare the median difference between the three groups. ${ }^{a} \mathrm{P}<0.05$ is statistically significant. IL, interleukin; VEGF, vascular endothelial growth factor; CCL-2, C-C motif chemokine ligand 2.

Table II. Expression (net MFI values) of IL-6, IL-10 and TNF- $\alpha$ in control $(n=20)$, pretreatment and ongoing-treatment groups $(\mathrm{n}=10$ each).

\begin{tabular}{lcccc}
\hline Cytokines & Control & Pretreatment & Ongoing-treatment & P-value \\
\hline IL-6 & $5.313 \pm 0.656$ & $16.13 \pm 7.905$ & $5.025 \pm 0.797$ & 0.3570 \\
IL-10 & $4.163 \pm 0.361$ & $3.875 \pm 0.582$ & $3.900 \pm 0.404$ & 0.8080 \\
TNF- $\alpha$ & $9.300 \pm 0.609$ & $9.525 \pm 1.570$ & $7.075 \pm 0.822$ & 0.1340 \\
\hline
\end{tabular}

Data values are expressed as mean \pm SEM. Kruskal Wallis test was conducted to compare the median difference between the three groups. IL, interleukin; MFI, median fluorescence intensity.

and TNF- $\alpha$ compared with the other groups. In contrast, the intensity of IL-10 was the lowest in the serum of the pretreatment group compared with the other two groups (Table II).

Correlation between the expression levels of cytokines and antinNav1.5-Ab in the serum samples. Correlation between the expression levels of antinNav1.5-Ab and the six cytokines among the three groups were analyzed using the Spearman correlation (Fig. 4). There was a significant positive correlation between the expression of antinNav1.5-Ab and IL-6 in the pre-treatment group. The other cytokines however, only showed poor to fair correlations when paired with antinNav1.5-Ab in the pretreatment group. In the ongoing-treatment group, the correlation between VEGF and the expression of antinNav1.5-Ab had a statistically significant inverse relationship. The other 5 cytokines, IL-8, IL-6, CCL2, IL-10 and TNF- $\alpha$ demonstrated good to poor correlations when paired with antinNav1.5-Ab in the ongoing-treatment group. However, these correlations were not significant. In the control group, all the cytokines exhibited poor to fair non-significant correlations with antinNav1.5-Ab.

\section{Discussion}

The metastatic potential of breast cancer cells increases with the degree of cell aggressiveness (32). Cells that originate from metastatic tumors are known to have unique genetic alterations to maintain their malignant characteristics, such as the ability to invade and metastasize (32). These genetic alterations may lead to the development of unique markers or antigens that contribute to the progression of metastases (32). The involvement of VGSCs was attributed to the metastatic capacity of breast cancer cells (23). Studies have demonstrated that the aberrant expression of a member of the VGSC family, Nav1.5 and its neonatal splice variant, nNav1.5, has established a mechanism to support the advancement of the disease (10-12). nNav1.5 has demonstrated onco-foetal upregulation in highly metastatic breast cancer cells as established in various in vitro $(23,24)$ and in vivo (26) studies. However, clinical studies involving nNav1.5 expression are only limited to breast tissues and biopsy samples $(12,21)$. Hence, the present study used serum samples collected from patients with breast cancer to discover the presence of antinNav1.5-Ab that were naturally present in the samples. The assessment of antinNav1.5-Ab in the present study reflected the expression level of nNav1.5 antigen carried by the circulating cancer cells present within the body systems of the participants.

As predicted, in the current study the serum samples of the breast cancer pre-treatment group exhibited the highest antinNav1.5-Ab expression compared with the other two groups tested. The pretreatment group did not receive any prior treatment, which contributed to the high level of antibodies present in the samples which may be due to the uninterrupted expression of nNav1.5 antigen carried by the metastatic or circulating breast cancer cells. The presence of antinNav1.5-Ab detected in the serum in the present study indicated the vulnerability and immunogenicity of the protein towards the actions of the immune system. The expression of antinNav1.5-Ab in the controls in the present study was somewhat surprising and indicated the natural presence of antinNav1.5-Ab in the serum of healthy female participants. Yamaci et al (21) demonstrated that some healthy epithelial breast ductal cells showed nNav1.5 immunoreactivity to a certain degree compared with breast cancer biopsies. In addition, in the aforementioned study immunoreactivity-staining was performed and the findings indicated that nNav1.5 expression appeared diffuse and less 
Control
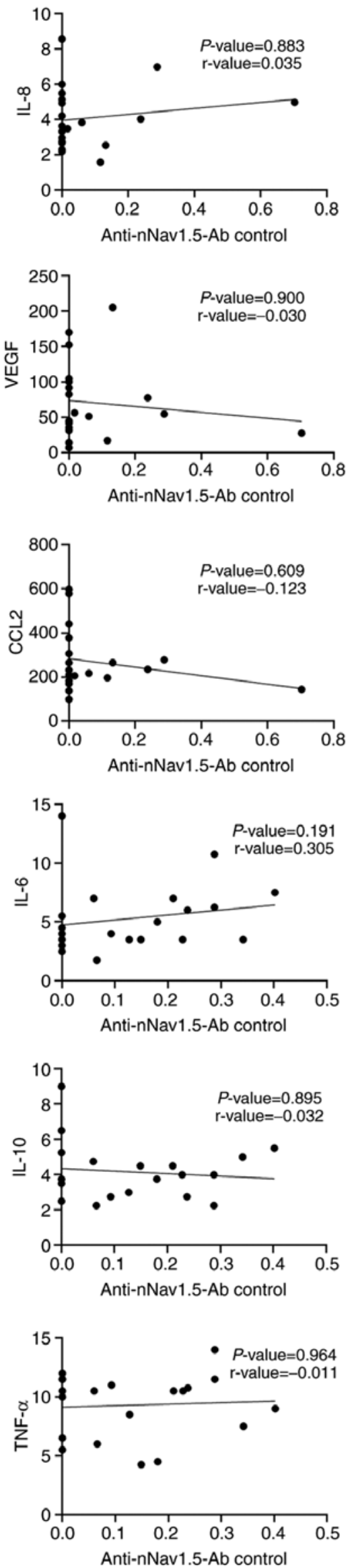

Pre-treatment
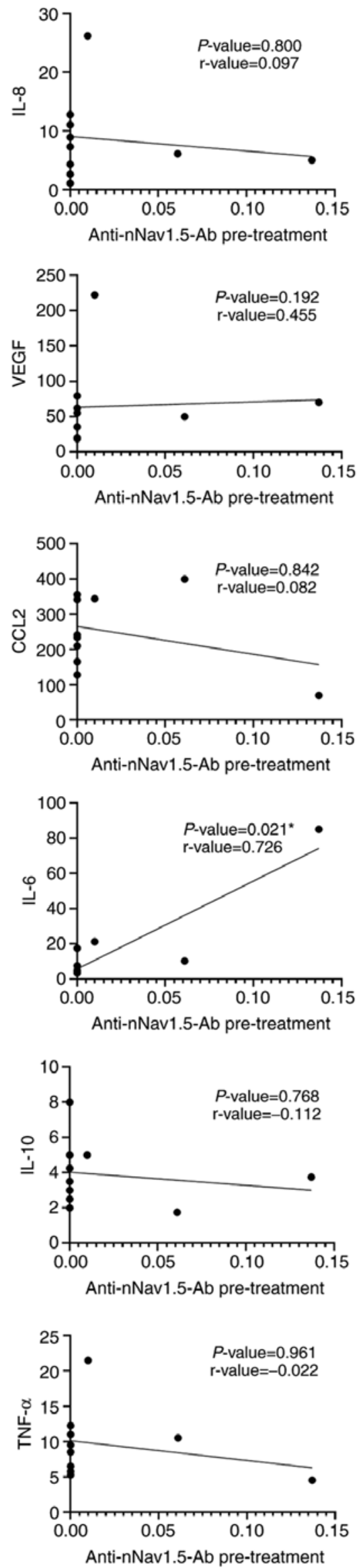

Ongoing-treatment
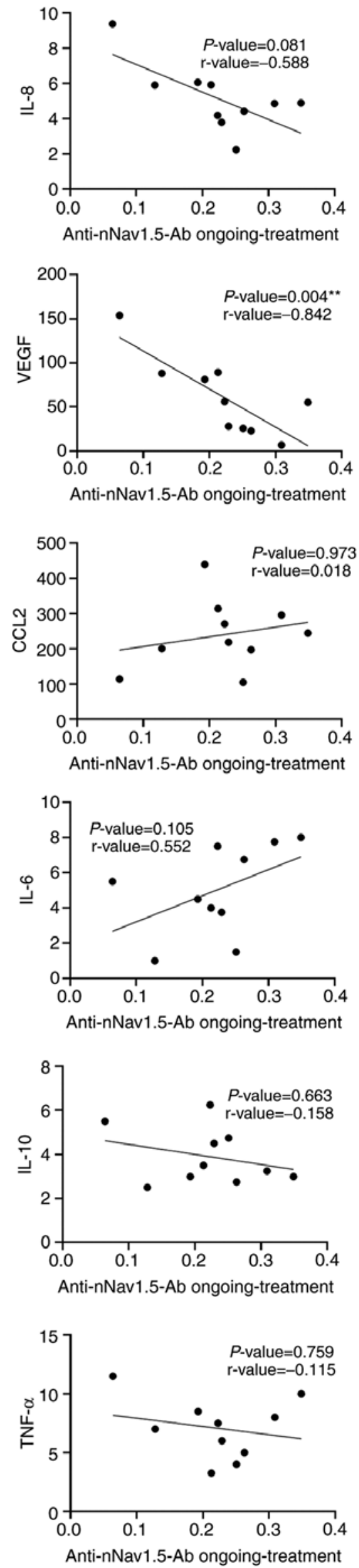

Figure 4. Correlation between cytokines and antinNav1.5-Ab expression. Correlations between antinNav1.5-Ab expression and cytokine levels of IL-8, VEGF, CCL2, IL-6, IL-10 and TNF- $\alpha$ among the three groups, control $(n=20)$, pre-treatment $(n=10)$ and ongoing-treatment $(n=10) .{ }^{*} \mathrm{P}=0.01-0.049,{ }^{* *} \mathrm{P} \leq 0.01 . \mathrm{IL}$, interleukin; VEGF, vascular endothelial growth factor; CCL-2, C-C motif chemokine ligand 2; antineonatal Nav1.5 antibodies (antinNav1.5-Ab). 
intense in healthy breast tissues compared with breast cancer tissues.

Metastatic breast disease can be treated or controlled by implementing various treatment strategies to achieve long-lasting remission and potentially cure (33). Breast cancer patients are often treated with chemotherapy $(34,35)$, radiation therapy (36), hormonal therapy (37), molecular therapy (38), immunotherapy (39) and surgery, such as mastectomy (40). In the present study, the expression of antinNav1.5-Ab in the serum of the ongoing-treatment group was much lower compared with the pre-treatment group. The lower expression of antinNav1.5-Ab in the ongoing treatment group in the present study was consistent with the declined state of metastasis due to treatment. Hence, it can be postulated that patients receiving treatment have a more stable disease control with respect to the downregulation of metastases compared with those who have not received any treatment, which may reflect the reduced state of $\mathrm{nNav1.5}$ expression that favors metastasis. However, there is another perspective that suggests that breast cancer treatment may reduce the expression of antibodies produced against nNav1.5, indicating the side effects of therapy that require further investigation. A study by Evans et al (41) found that the combination of two forms of treatment, which includes chemotherapy, radiotherapy or hormonal therapy tends to reduce the expression of autoantibodies produced against tumor-associated antigens in patients with breast cancer. The difference in the pattern of antinNav1.5-Ab expression demonstrated between the three groups in the present study highlights the capacity of this antibody to act as an immunosurveillance marker during breast cancer treatment that can be used to monitor the efficacy of breast cancer treatment in eliminating breast cancer metastasis.

CD25 is the $\alpha$-chain of the heterotrimeric IL-2 receptor complex (42). According to Lundin et al (43), in comparison to the $\gamma$-chain, considerable amounts of the $\alpha$-chain can be found in serum. Hence, serum samples are reliable biological sources that can be used to measure the concentration of CD25 (44). CD25 is a well-established T cell activation marker that is widely used to measure Treg cell expression levels (44). Established immune evasion has been identified as one of the critical factors for tumor development and cancer progression (27). Over time, Treg cells have been studied in association with tumor progression. Studies have demonstrated that Treg cells promote the initiation and progression of tumors as well as the induce neo-angiogenesis (45-47). In the case of cancer, Treg cells compromise the antitumor response by reducing the efficiency of $\mathrm{T}$ cells. For instance in a study by Shang et al (48), there was increased Treg tumor infiltration and a low T-effector cell ratio was associated with poor prognosis in cases of solid tumors including cervical, renal, melanomas, and breast cancers. CD25 was the first surface marker identified to distinguish Treg cells (49) prior to the discovery of their master regulator known as Fork-head box P3 (Foxp3). C-C chemokine receptor 4 on Treg cells allow the recruitment of cells to the microenvironment of the tumor after the release of CCL22 by tumor associated macrophages (50). Studies have found that Treg cells impart immunosuppression by blocking the production of interferon-gamma (IFN- $\gamma$ ) and IL-2 cells by T-effector cells $(51,52)$. In the present study as predicted, the CD25 concentration was the highest in the pretreatment group followed by the control and ongoing treatment groups. The elevated presence of metastases in the pretreatment group indicated by antinNav1.5-Ab expression appeared to be validated by the high concentration of CD25, representing the Treg population. This finding is consistent with a study by Nishikawa et al (53) in which it was assumed that the promotion of metastases could result from the immunosuppressive effects of Treg cells. However, in the present study the CD25 concentration in the serum of the ongoing-treatment group was lower compared with the pretreatment and control groups, highlighting the effects of breast cancer treatment on metastatic breast cancer by indirectly lowering the expression of Treg cells. Treg cells have been found to have been reduced following a single intravenous infusion of daclizumab in patients with metastatic breast cancer (54). In 2011, an in vivo mouse mesothelioma model study found that the repopulation of tumor cells between cycles of chemotherapy was inhibited by the depletion of Treg cells (55). Lissoni et al (56) reported that the induction of chemotherapy (irrespective of the regimen) led to a decrease in number of Treg cells in patients with stable disease or tumor regression as compared with those with progressive disease which suggested that metastatic cancer disease is characterized by an upregulation in the Treg cell count.

The presence of metastases may interfere with the expression levels of the immune system's vital components, such as cytokines (57). The assessment of the levels of pro-inflammatory and antiinflammatory cytokines associated with breast cancer metastases provides a picture of the progression of cancer in the body system (57). Cytokines are secreted proteins that may be induced to mediate intercellular communication within the immune system (57). Cytokines may be grouped into several categories, such as TNF, IL, IFN, chemokines and colony-stimulating factors (57). The cytokine levels of IL-8, VEGF, CCL2, IL-6, IL-10 and TNF- $\alpha$ were examined in the present study on the basis of their association with metastases for breast cancer.

In the present study, the pre-treatment group had the highest expression levels of IL-8, IL- 6 and TNF- $\alpha$ compared to the control and ongoing-treatment groups. These findings indicated the presence of metastases among pre-treatment patients with breast cancer as these 3 cytokines are well-known contributors to the progression of metastases. IL- 8 is generally associated with lymph node metastases (58) and it promotes breast cancer stem cell activity via its cognate receptors, chemokine receptors (CXCR)1/2 (59) whereas IL-6 promotes metastases by favoring signal transducer and activator of transcription 3 (STAT3) pathway activation (60). The ligation of IL-6 to its receptor stimulates Janus kinase (JAK) tyrosine kinases leads to the phosphorylation of STAT3 (61). The homodimerization and entrance of STAT3 in cancer cells modulates the proliferation, survival and transformation (61). Increased level of IL-6 is often associated with poor survival and prognosis in patients with breast cancer $(62,63)$. The positive correlation between the expression of IL-6 and antinNav1.5-Ab in the pre-treatment group in the present study further validates that the provocation of the immune system to produce antibodies against nNav1.5 happens in parallel with the progression of metastases reflected by the upregulation of IL-6. On the other hand, TNF- $\alpha$ enhances the process of metastases by 
promoting the inflammatory microenvironment and enhances the expressions of matrix metalloproteinases (64), IL-8 (65) and CXCR (66). In the present study, the pretreatment group conversely had the lowest expression of IL-10. IL-10 is a pleiotropic immunoregulatory cytokine that exhibits both pro- and antitumor activities (67). In spite of the contrasting opinions regarding the IL-10 expression in breast cancer (67), the findings of the present study are in concert of those of Li et al (68). Li et al (68) discovered that the low expression of IL-10 leads to poor survival outcome in patients with breast cancer and is also associated with disease-free survival (recovered from breast cancer) which explains why the pre-treatment group in the present study had the lowest expression of IL-10. In summary, the imbalance between metastasis-favoring (IL-8, IL- 6 and TNF- $\alpha$ ) and metastasis-opposing cytokines (IL-10) is beneficial to the progression of metastases. Based on the findings of the present study, antinNav1.5-Ab expression and its association with metastasis-favoring cytokines in the pretreatment group, antinNav1.5-Ab may be a metastasis marker with immune-surveillance properties capable of monitoring the progression of metastasis among patients with breast cancer.

The ongoing-treatment group in the present study had the lowest expression levels of CCL2, VEGF, IL-6 and TNF- $\alpha$ compared with the other tested groups. The low expressions of these cytokines indicated that the breast cancer therapies conducted had productively overcome metastases to a certain extent and may be able to provide a good treatment prognosis.

CCL2, also known as monocyte chemoattractant protein-1 (MCP-1) is implicated in the progression of cancer where it enhances cell invasion via the modulation of the MAP kinase pathway (MCP-1-CCR2 axis) (69). CCL2 binds to its cognate receptor, CCR2 and promotes multiple pro-tumorigenic roles which include intravasation, extravasation and angiogenesis (70) and formation of metastasis foci (71). The upregulation transcription factor, Twist1 in human mammary cells favors angiogenesis via the induction of CCL2 (72). Conversely, the downregulation of CCL2 leads to an improved outcome and lower metastasis in patients with breast cancer as suggested by Qian et al (73).

VEGF is a pro-angiogenic protein that triggers the 'angiogenic switch' to promote the formation of endothelial-mediated blood vessels (57). Tumor development is often associated with a decrease in the oxygen tension which is mainly due to poor vascularization (57). To overcome this, the process of tumor angiogenesis is stimulated to provide nutrients and oxygen for the tumor sites that have reached a state of hypoxia (57). Nav1.5, the adult isoform of nNav1.5 was discovered as one of the significant VGSC $\alpha$ isoforms (91\% of total VGSC $\alpha$ ) present in HUVEC cells (74). In 2011, Andrikopoulos et al (74), found that Nav1.5 potentiates angiogenesis via VEGF-induced ERK1/2 activation through the protein kinase $\mathrm{C} \alpha-\mathrm{B}-\mathrm{RAF}$ signaling axis. In addition, the aforementioned study demonstrated that the potentiation takes place through the modulation of VEGF-induced HUVEC depolarization and by altering the kinetics of calcium ions (74). The involvement of calcium ions in different phases of angiogenesis and the modulation of VEGF was highlighted in another study (75). The inflow of calcium ions via the reverse mode sodium-calcium exchanger is necessary for the activation of PKC, VEGF-induced ERK1/2 phosphorylation and the downstream role of endothelial cells in angiogenesis (76). The findings of the current study may indicate that the neonatal isoform also has the capacity to initiate VEGF-induced endothelial angiogenesis as the increased production of antibodies targeting nNav1.5 was parallel to the decrease in the level of VEGF.

As a metastatic preferring cytokine, it was anticipated that IL- 8 will be reduced after treatment in the present study. Surprisingly, IL-8 was upregulated in the serum samples of the ongoing treatment group in the present study. This is in concert with the findings of Ginestier et al (77). The aforementioned study postulated that chemotherapy-induced cell injury may lead to the upregulation of IL-8 which could stimulate cancer stem cells to replicate to resume the progression of cancer (77). Another study also demonstrated the production of IL- 8 by injured cancer cells, which reinitiates cancer progression (78). The findings of this study were in agreement with those of Ginestier et al (77).

The limitations of the present study included the absence of a positive control to validate the antinNav1.5-Ab indirect ELISA assay and the absence of a standard curve to evaluate the concentration of antinNav1.5-Ab in the serum of patients with breast cancer. In the upcoming studies, the availability of these positive controls and standards will further validate the efficiency of the in-house assay.

In conclusion, the detection of antinNav1.5-Ab in the serum of the present study clearly indicated the crosstalk between breast cancer metastasis and immune cells, adding another layer of complexity to the understanding of metastasis formation while opening new therapeutic opportunities for patients with breast cancer. The consistency of antinNav1.5-Ab expression with the manifestation of other immune system components such as Tregs and cytokines in the present study provides a promising opportunity to promote the function of nNav1.5 as a metastasis marker as well as an immune-surveillance tool in breast cancer therapy as such therapy does affect the expression of antinNav1.5-Ab.

\section{Acknowledgements}

The authors would like to thank the medical officers, Dr Norshahida Mohamed Noordin, Dr Nur'atikah Adnan, Dr Lee Lih Shin and nurses, Sister Roslaini Che Romli and Siti Eshah Harun from the Breast Cancer Awareness and Research Unit (BestARi) under Hospital Universiti Sains Malaysia (HUSM) for their corporation during patient recruitment. The authors would also like to thank Dr Wong Weng Kin from the School of Health Sciences for his guidance on planning the in-house indirect ELISA.

\section{Funding}

The present study was funded by the Research University Individual (RUI) grant (grant no. 1001/PPSK/8012275) and the Science Fund, Ministry of Science, Technology, Innovation, Malaysia (MOSTI) (grant no. 06-01-05-SF0844).

\section{Availability of data and materials}

The datasets used and/or analyzed during the current study are available from the corresponding author on reasonable request. 


\section{Authors' contributions}

HR made substantial contributions to the conception and design, acquisition, analysis and interpretation of data and in writing the manuscript. NSR was involved in the interpretation of the data and the statistical analyses. TADAA was involved in the acquisition of data and recruitment of breast cancer patients. NFM contributed to the idea for the conception and design, interpretation of data, writing the manuscript, revising it critically for important intellectual content and obtained funding for the peptide solution. MMY and WZWZ were extensively involved in the patient recruitment, blood withdrawal, patient diagnosis, interpretation of the data, revising and editing the manuscript critically for intellectual content. NAA was involved in both conception and idea generation, drafting the manuscript, revising it critically for important intellectual content and involved in analysis and interpretation of data. WEMF was involved in conception and idea generation, ethical defense, acquisition of data, interpretation of data, drafting the manuscript, revising it critically for important intellectual content, giving final approval of the version to be published and overall funding for the study.

\section{Ethics approval and consent to participate}

Ethical approval for this study was granted by the Human Research Ethics Committee of USM (JEPeM; Kubang Kerian, Malaysia) (approval no. USM/JEPeM/18100518). Written informed consents were obtained from all subjects prior to blood collection.

\section{Patient consent for publication}

Not applicable.

\section{Competing interests}

The authors declare that they have no competing interests.

\section{References}

1. International Agency for Research on Cancer (IARC). GLOBOCAN report. 2018. Retrieved from https://www.iarc.fr/.

2. Centers for Disease Control and Prevention (CDC), Division of Cancer Prevention and Control, Breast Cancer Statistics. 2019. Retrieved from https://www.cdc.gov/cancer/breast/basic_ info/index.htm.

3. Feng Y, Spezia M, Huang S, Yuan C, Zeng Z, Zhang L, Ji X, Liu W, Huang B, Luo W, et al: Breast cancer development and progression: Risk factors, cancer stem cells, signaling pathways, genomics, and molecular pathogenesis. Genes Dis 5: 77-106, 2018.

4. Sharma GN, Dave R, Sanadya J, Sharma P and Sharma KK: Various types and management of breast cancer: An overview. J Adv Pharm Technol Res 1: 109-126, 2010.

5. Akram M, Iqbal M, Daniyal M and Khan AU: Awareness and current knowledge of breast cancer. Biol Res 50: 33-33, 2017.

6. van Zijl F, Krupitza G and Mikulits W: Initial steps of metastasis: Cell invasion and endothelial transmigration. Mutat Res 728 : 23-34, 2011.

7. Seyfried TN and Huysentruyt LC: On the origin of cancer metastasis. Crit Rev Oncog 18: 43-73, 2013.

8. Djamgoz MB, Coombes RC and Schwab A: Ion transport and cancer: From initiation to metastasis. Philos Trans R Soc Lond B Biol Sci 369: 20130092, 2014.
9. Koltai T: Cancer: Fundamentals behind $\mathrm{pH}$ targeting and the double-edged approach. Onco Targets Ther 9: 6343-6360, 2016.

10. Brackenbury WJ: Voltage-gated sodium channels and metastatic disease. Channels 6: 352-361, 2012.

11. Roger S, Besson P and Le Guennec JY: Involvement of a novel fast inward sodium current in the invasion capacity of a breast cancer cell line. Biochim Biophys Acta 1616: 107-111, 2003.

12. Fraser SP, Diss JK, Chioni AM, Mycielska ME, Pan H, Yamaci RF, Pani F, Siwy Z, Krasowska M, Grzywna Z, et al: Voltage-gated sodium channel expression and potentiation of human breast cancer metastasis. Clin Cancer Res 11: 5381-5389, 2005.

13. Onganer PU, Seckl MJ and Djamgoz MB: Neuronal characteristics of small-cell lung cancer. Br J Cancer 93: 1197-1201, 2005.

14. Diaz D, Delgadillo DM, Hernández-Gallegos E, Ramírez-Domínguez ME, Hinojosa LM, Ortiz CS, Berumen J, Camacho $\mathrm{J}$ and Gomora JC: Functional expression of voltage-gated sodium channels in primary cultures of human cervical cancer. J Cell Physiol 210: 469-478, 2007.

15. Gao R, Shen Y, Cai J, Lei M and Wang Z: Expression of voltage-gated sodium channel $\alpha$ subunit in human ovarian cancer. Oncol Rep 23: 1293-1299, 2010.

16. Guzel RM, Ogmen K, Ilieva KM, Fraser SP and Djamgoz MBA: Colorectal cancer invasiveness in vitro: Predominant contribution of neonatal Nav1.5 under normoxia and hypoxia. J Cell Physiol 234: 6582-6593, 2019.

17. House CD, Vaske CJ, Schwartz AM, Obias V, Frank B, Luu T, Sarvazyan N, Irby R, Strausberg RL, Hales TG, et al: Voltage-gated $\mathrm{Na}^{+}$channel $S C N 5 A$ is a key regulator of a gene transcriptional network that controls colon cancer invasion. Cancer Res 70: 6957-6967, 2010.

18. Ma RSY, Kayani K, Whyte-Oshodi D, Whyte-Oshodi A, Nachiappan N, Gnanarajah S and Mohammed R: Voltage gated sodium channels as therapeutic targets for chronic pain. J Pain Res 12: 2709-2722, 2019.

19. Utrilla RG, Nieto-Marín P, Alfayate S, Tinaquero D, Matamoros M, Pérez-Hernández M, Sacristán S, Ondo L, de Andrés R, Díez-Guerra FJ, et al: Kir2. 1-Nav1. 5 channel complexes are differently regulated than Kir2. 1 and Nav1. 5 channels alone. Front Physiol 8: 903, 2017.

20. Onkal R, Mattis JH, Fraser SP, Diss JK, Shao D, Okuse K and Djamgoz MB: Alternative splicing of Nav1.5: An electrophysiological comparison of 'neonatal' and 'adult' isoforms and critical involvement of a lysine residue. J Cell Physiol 216: 716-726, 2008.

21. Yamaci RF, Fraser SP, Battaloglu E, Kaya H, Erguler K, Foster CS and Djamgoz MBA: Neonatal Nav1.5 protein expression in normal adult human tissues and breast cancer. Pathol Res Pract 213: 900-907, 2017

22. Ben-Porath I, Thomson MW, Carey VJ, Ge R, Bell GW, Regev A and Weinberg RA: An embryonic stem cell-like gene expression signature in poorly differentiated aggressive human tumors. Nat Genet 40: 499-507, 2008

23. Brackenbury WJ, Chioni AM, Diss JK and Djamgoz MB: The neonatal splice variant of Nav1.5 potentiates in vitro invasive behaviour of MDA-MB-231 human breast cancer cells. Breast Cancer Res Treat 101: 149-160, 2007.

24. Isbilen B, Fraser SP and Djamgoz MB: Docosahexaenoic acid (omega-3) blocks voltage-gated sodium channel activity and migration of MDA-MB-231 human breast cancer cells. Int J Biochem Cell Biol 38: 2173-2182, 2006.

25. Erdogan MA and Ozpolat B: Targeting of Voltage-gated sodium channel $\mathrm{Na}_{\mathrm{v}} 1.5$ inhibits cell proliferation and colony formation in breast and ovarian cancer cells. Cancer Res: 73 (8 Suppl), 2013 doi: 10.1158/1538-7445.AM2013-514.

26. Nelson M, Yang M, Millican-Slater R and Brackenbury WJ Nav1.5 regulates breast tumor growth and metastatic dissemination in vivo. Oncotarget 6: 32914-32929, 2015.

27. Hanahan D and Weinberg RA: Hallmarks of cancer: The next generation. Cell 144: 646-674, 2011.

28. Norsa'adah B: Univariable Analyses Using IBM SPSS statistics version 20.0, Universiti Sains Malaysia, Malaysia, 2013.

29. George D and Mallery P: Chapter 7: Descriptive Statistics. In: SPSS for Windows Step by Step: a Simple Study Guide and Reference 17.0 Update. 10th edition. Pearson, Boston, MA, 2010.

30. Schmider E, Ziegler M, Danay E, Beyer L and Bühner M: Is it really robust? Reinvestigating the robustness of ANOVA against violations of the normal distribution. Methodology 6: 147-151,2010.

31. Breen EJ, Tan W and Khan A: The statistical value of raw fluorescence signal in Luminex xMAP Based Multiplex Immunoassays. Sci Rep 6: 26996-26996, 2016. 
32. Rhana P, Trivelato RRJ, Beirao PSL, Cruz JS and Rodrigues ALP: Is there a role for voltage-gated $\mathrm{Na}^{+}$channels in the aggressiveness of breast cancer? Braz J Med Biol Res 50: e6011, 2017.

33. Pagani O, Senkus E, Wood W, Colleoni M, Cufer T, Kyriakides S, Costa A, Winer EP and Cardoso F; ESO-MBC Task Force: International guidelines for management of metastatic breast cancer: Can metastatic breast cancer be cured? J Natl Cancer Inst 102: 456-463, 2010.

34. Waks AG and Winer EP: Breast cancer treatment: A review. JAMA 321: 288-300, 2019.

35. Fujii T, Le Du F, Xiao L, Kogawa T, Barcenas CH, Alvarez RH, Valero V, Shen Y and Ueno NT: Effectiveness of an adjuvant chemotherapy regimen for early-stage breast cancer: A systematic review and network meta-analysis. JAMA Oncol 1: 1311-1318, 2015.

36. Krug D: Adjuvant radiotherapy for breast cancer: More than meets the eye. Breast Care (Basel) 15: 109-111, 2020.

37. Tremont A, Lu J and Cole JT: Endocrine therapy for early breast cancer: Updated review. Ochsner J 17: 405-411, 2017.

38. Munagala R, Aqil F and Gupta RC: Promising molecular targeted therapies in breast cancer. Indian J Pharmacol 43: 236-245, 2011.

39. U.S Food and Drug Administration (FDA). FDA approves Atezolizumab for PD-L1 positive unresectable locally advanced or metastatic triple-negative breast cancer. Retrieved from https://www.fda.gov/drugs/drug-approvals-and-databases/fda-approves-atezolizumab-pd-11-positive-unresectable-lo cally-advanced-or-metastatic-triple-negative.

40. Zurrida S, Bassi F, Arnone P, Martella S, Del Castillo A, Ribeiro Martini R, Semenkiw ME and Caldarella P: The changing face of mastectomy (from Mutilation to Aid to Breast Reconstruction). Int J Surg Oncol 2011: 980158, 2011.

41. Evans RL, Pottala JV, Nagata S and Egland KA: Longitudinal autoantibody responses against tumor-associated antigens decrease in breast cancer patients according to treatment modality. BMC Cancer 18: 119, 2018.

42. Witkowska AM: On the role of sIL-2R measurements in rheumatoid arthritis and cancers. Mediators Inflamm 2005: 121-130, 2005

43. Lundin K, Tuukkanen AM, Jansson C, Nordström T and Lindqvist C: No soluble common cytokine receptor gamma chain (gamma(c)) in activated human lymphocyte cultures-comparison with soluble IL-2Ralpha. Immunol Lett 82: 235-240, 2002.

44. Gotoh Y, Okamoto Y, Uemura O, Mori N, Tanaka S, Ando T and Nishida M: Determination of age-related changes in human soluble interleukin 2 receptor in body fluids of normal subjects as a control value against disease states. Clin Chim Acta 289: 89-97, 1999.

45. Mougiakakos D, Choudhury A, Lladser A, Kiessling R and Johansson CC: Regulatory T cells in cancer. Adv Cancer Res 107: $57-117,2010$

46. Dwarakanath BS, Farooque A and Gupta S: Targeting regulatory $\mathrm{T}$ cells for improving cancer therapy: Challenges and prospects Cancer Rep (Hoboken) 1: e21105, 2018.

47. Halvorsen EC, Hamilton MJ, Young A, Wadsworth BJ, LePard NE, Lee HN, Firmino N, Collier JL and Bennewith KL: Maraviroc decreases CCL8-mediated migration of $\mathrm{CCR}^{+}{ }^{+}$regulatory $\mathrm{T}$ cells and reduces metastatic tumor growth in the lungs. Oncoimmunology 5: e1150398, 2016.

48. Shang B, Liu Y, Jiang SJ and Liu Y: Prognostic value of tumor-infiltrating FoxP $3^{+}$regulatory $\mathrm{T}$ cells in cancers: A systematic review and meta-analysis. Sci Rep 5: 15179, 2015.

49. Sakaguchi S, Sakaguchi N, Asano M, Itoh M and Toda M: Immunologic self-tolerance maintained by activated $\mathrm{T}$ cells expressing IL-2 receptor alpha-chains (CD25). Breakdown of a single mechanism of self-tolerance causes various autoimmune diseases. J Immunol 155: 1151-1164, 1995.

50. Wei S, Kryczek I, Edwards RP, Zou L, Szeliga W, Banerjee M, Cost M, Cheng P, Chang A, Redman B, et al: Interleukin-2 administration alters the $\mathrm{CD} 4^{+} \mathrm{FOXP} 3^{+} \mathrm{T}$-cell pool and tumor trafficking in patients with ovarian carcinoma. Cancer Res 67 7487-7494, 2007

51. Liu S, Sun X, Luo J, Zhu H, Yang X, Guo Q, Song Y and Sun X: Effects of radiation on $\mathrm{T}$ regulatory cells in normal states and cancer: Mechanisms and clinical implications. Am J Cancer Res 5: 3276-3285, 2015.

52. Yu P, Lee Y, Liu W, Krausz T, Chong A, Schreiber H and Fu YX: Intratumor depletion of $\mathrm{CD}^{+}$cells unmasks tumor immunogenicity leading to the rejection of late-stage tumors. J Exp Med 201: 779-791, 2005.

53. Nishikawa $H$, Kato T, Tanida K, Hiasa A, Tawara I, Ikeda $H$, Ikarashi Y, Wakasugi H, Kronenberg M, Nakayama T, et al: $\mathrm{CD} 4^{+} \mathrm{CD} 25^{+} \mathrm{T}$ cells responding to serologically defined autoantigens suppress antitumor immune responses. Proc Natl Acad Sci USA 100: 10902-10906, 2003.
54. Rech AJ and Vonderheide RH: Clinical use of Anti-CD25 antibody daclizumab to enhance immune responses to tumor antigen vaccination by targeting regulatory T cells. Ann N Y Acad Sci 1174: 99-106, 2009.

55. Wu L, Yun Z, Tagawa T, Rey-McIntyre K, Anraku M and Perrot M: Tumor cell repopulation between cycles of chemotherapy is inhibited by regulatory T-cell depletion in a murine mesothelioma model. J Thorac Oncol 6: 1578-1586, 2011.

56. Lissoni P, Brivio F, Fumagalli L, Messina G, Meregalli S, Porro G, Rovelli F, Vigorè L, Tisi E and D'Amico G: Effects of the conventional antitumor therapies surgery, chemotherapy, radiotherapy and immunotherapy on regulatory $\mathrm{T}$ lymphocytes in cancer patients. Anticancer Res 29: 1847-1852, 2009.

57. Esquivel-Velázquez M, Ostoa-Saloma P, Palacios-Arreola MI, Nava-Castro KE, Castro JI and Morales-Montor J: The role of cytokines in breast cancer development and progression. J Interferon Cytokine Res 35: 1-16, 2015.

58. Benoy IH, Salgado R, Van Dam P, Geboers K, Van Marck E, Scharpé S, Vermeulen PB and Dirix LY: Increased serum interleukin- 8 in patients with early and metastatic breast cancer correlates with early dissemination and survival. Clin Cancer Res 10: 7157, 2004.

59. Singh JK, Farnie G, Bundred NJ, Simões BM, Shergill A, Landberg G, Howell SJ and Clarke RB: Targeting CXCR1/2 significantly reduces breast cancer stem cell activity and increases the efficacy of inhibiting HER2 via HER2-dependent and -independent mechanisms. Clin Cancer Res 19: 643-656, 2013.

60. Chang Q, Bournazou E, Sansone P, Berishaj M, Gao SP, Daly L, Wels J, Theilen T, Granitto S, Zhang X, et al: The IL-6/JAK/Stat 3 feed-forward loop drives tumorigenesis and metastasis. Neoplasia 15: 848-862, 2013.

61. Masjedi A, Hashemi V, Hojjat-Farsangi M, Ghalamfarsa G, Azizi G, Yousefi M and Jadidi-Niaragh F: The significant role of interleukin- 6 and its signaling pathway in the immunopathogenesis and treatment of breast cancer. Biomed Pharmacother 108: $1415-1424,2018$

62. Lin S, Gan Z, Han K, Yao Y and Min D: Interleukin-6 as a prognostic marker for breast cancer: A meta-analysis. Tumori 101: 535-541, 2015.

63. Shibayama O, Yoshiuchi K, Inagaki M, Matsuoka Y, Yoshikawa E, Sugawara Y, Akechi T, Wada N, Imoto S, Murakami K, et al: Association between adjuvant regional radiotherapy and cognitive function in breast cancer patients treated with conservation therapy. Cancer Med 3: 702-709, 2014.

64. Wolczyk D, Zaremba-Czogalla M, Hryniewicz-Jankowska A, Tabola R, Grabowski K, Sikorski AF and Augoff K: TNF- $\alpha$ promotes breast cancer cell migration and enhances the concentration of membrane-associated proteases in lipid rafts. Cell Oncol (Dordr) 39: 353-363, 2016.

65. Osawa Y, Nagaki M, Banno Y, Brenner DA, Asano T, Nozawa Y, Moriwaki $\mathrm{H}$ and Nakashima S: Tumor necrosis factor alpha-induced interleukin-8 production via NF-kappaB and phosphatidylinositol 3-kinase/Akt pathways inhibits cell apoptosis in human hepatocytes. Infect Immun 70: 6294-6301, 2002.

66. Kulbe H,Hagemann T, Szlosarek PW, Balkwill FR and Wilson JL: The inflammatory cytokine tumor necrosis factor-alpha regulates chemokine receptor expression on ovarian cancer cells. Cancer Res 65: 10355-10362, 2005.

67. Sheikhpour E, Noorbakhsh P, Foroughi E, Farahnak S, Nasiri R and Neamatzadeh H: A survey on the role of interleukin-10 in breast cancer: A narrative. Rep Biochem Mol Biol 7: 30-37, 2018.

68. Li Y, Gao P, Yang J, Yu H, Zhu Y and Si W: Relationship between IL-10 expression and prognosis in patients with primary breast cancer. Tumour Biol 35: 11533-11540, 2014

69. Dutta P, Sarkissyan M, Paico K, Wu Y and Vadgama JV: MCP-1 is overexpressed in triple-negative breast cancers and drives cancer invasiveness and metastasis. Breast Cancer Res Treat 170. 477-486, 2018.

70. Lim SY, Yuzhalin AE, Gordon-Weeks AN and Muschel RJ: Targeting the CCL2-CCR2 signaling axis in cancer metastasis. Oncotarget 7: 28697-28710, 2016.

71. Kitamura T, Qian BZ, Soong D, Cassetta L, Noy R, Sugano G, Kato Y, Li J and Pollard JW: CCL2-induced chemokine cascade promotes breast cancer metastasis by enhancing retention of metastasis-associated macrophages. J Exp Med 212: 1043-1059, 2015. 
72. Low-Marchelli JM, Ardi VC, Vizcarra EA, van Rooijen N, Quigley JP and Yang J: Twist1 induces CCL2 and recruits macrophages to promote angiogenesis. Cancer Res 73: 662-671, 2013.

73. Qian BZ, Li J, Zhang H, Kitamura T, Zhang J, Campion LR, Kaiser EA, Snyder LA and Pollard JW: CCL2 recruits inflammatory monocytes to facilitate breast-tumor metastasis. Nature 475 222-225, 2011.

74. Andrikopoulos P, Fraser SP, Patterson L, Ahmad Z, Burcu H, Ottaviani D, Diss JK, Box C, Eccles SA and Djamgoz MB: Angiogenic functions of voltage-gated $\mathrm{Na}^{+}$channels in human endothelial cells: Modulation of vascular endothelial growth factor (VEGF) signaling. J Biol Chem 286: 16846-16860, 2011.

75. Fiorio Pla A and Munaron L: Functional properties of ion channels and transporters in tumor vascularization. Philos Trans R Soc Lond B Biol Sci 369: 20130103, 2014.

76. Andrikopoulos P, Baba A, Matsuda T, Djamgoz MBA, Yaqoob MM and Eccles SA: $\mathrm{Ca}^{+}$influx through reverse mode
$\mathrm{Na}^{+} / \mathrm{Ca} 2^{+}$exchange is critical for vascular endothelial growth factor-mediated extracellular signal-regulated kinase (ERK) $1 / 2$ activation and angiogenic functions of human endothelial cells. J Biol Chem 286: 37919-37931, 2011.

77. Ginestier C, Liu S, Diebel ME, Korkaya H, Luo M, Brown M, Wicinski J, Cabaud O, Charafe-Jauffret E, Birnbaum D, Guan JL, et al: CXCR1 blockade selectively targets human breast cancer stem cells in vitro and in xenografts. J Clin Invest 120: 485-497, 2010

78. Todorović-Raković $\mathrm{N}$ and Milovanović J: Interleukin-8 in breast cancer progression. J Interferon Cytokine Res 33: 563-570, 2013.

(i) $($ ) $९$ This work is licensed under a Creative Commons Attribution-NonCommercial-NoDerivatives 4.0 International (CC BY-NC-ND 4.0) License. 